NIKHEF-H/94-33

\title{
Tau Decays into Kaons in DELPHI ${ }^{1}$
}

\author{
W. Ruckstuhl \\ NIKHEF-H \\ P.O. Box 41882 \\ 1009 DB Amsterdam \\ The Netherlands
}

\begin{abstract}
Exclusive and inclusive branching ratios of several $\tau$ decays with kaons in the final state have been measured using data collected with the DELPHI detector. Charged kaons are tagged over a large momentum range by the Ring Imaging Cherenkov detector, and the secondary vertices of $K_{s}^{0}$ decays into charged pions are reconstructed. The result $\mathrm{BR}(\tau \rightarrow K \nu)=(0.85 \pm 0.18) \%$ is in agreement with the Standard Model prediction based on $\tau-\mu-e$ universality. The branching ratio $\mathrm{BR}\left(\tau \rightarrow K^{*} \nu\right)=(1.71 \pm 0.36) \%$ is a combination of two independent measurements in the $\pi^{ \pm} K_{s}^{0}$ and the $K^{ \pm} \pi^{0}$ mode. Also inclusive branching ratio were measured: $\mathrm{BR}(\tau \rightarrow K \nu \geq 0$ neutrals $)$ $=(1.54 \pm 0.24) \%, \mathrm{BR}(\tau \rightarrow K \nu \geq 1$ neutrals $)=(0.64 \pm 0.24) \%$ and $\operatorname{BR}\left(\tau \rightarrow K_{s}^{0} X^{ \pm} \nu\right)=(0.99 \pm 0.19) \%$. The uncertainties of all results are dominated by statistics.
\end{abstract}

\footnotetext{
${ }^{1}$ Presented at the 1994 Third Workshop on Tau Lepton Physics, Montreux, September 19-22, 1994.
} 


\title{
Tau Decays into Kaons in DELPHI
}

\author{
W. Ruckstuhl ${ }^{\mathrm{a}}$
}

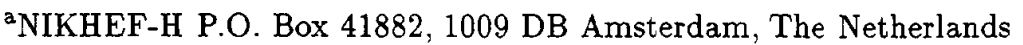

\begin{abstract}
Exclusive and inclusive branching ratios of several $\tau$ decays with kaons in the final state have been measured using data collected with the DELPHI detector. Charged kaons are tagged over a large momentum range by the Ring Imaging Cherenkov detector, and the secondary vertices of $K_{s}^{-0}$ decays into charged pions are reconstructed. The result $\operatorname{BR}(\tau \rightarrow K \nu)=(0.85 \pm 0.18) \%$ is in agreement with the Standard Model prediction based on $\tau-\mu-e$ universality. The branching ratio $\operatorname{BR}\left(\tau \rightarrow K^{*} \nu\right)=(1.71 \pm 0.36) \%$ is a combination of two independent measurements in the $\pi^{ \pm} K_{s}^{0}$ and the $K^{ \pm} \pi^{0}$ mode. Also inclusive branching ratio were measured: $\operatorname{BR}(\tau \rightarrow K \nu \geq$ 0 neutrals $)=(1.54 \pm 0.24) \%, \mathrm{BR}(\tau \rightarrow K \nu \geq 1$ neutrals $)=(0.64 \pm 0.24) \%$ and $\mathrm{BR}\left(\tau \rightarrow K_{s}^{0} X^{ \pm} \nu\right)=(0.99 \pm$ $0.19) \%$. The uncertainties of all results are dominated by statistics.
\end{abstract}

\section{Introduction}

In the last few years, the measurements of $\tau$ branching ratios have reached a new level of precision. This improvement is mainly due to the large $\tau$ pair samples available at LEP and CESR and to new advanced detectors. Such advanced techniques, especially particle identification, are necessary to measure the low branching ratios of $\tau$ decays with kaons in the final state. I present here a study of such decays using the DELPHI detector at LEP. In DELPHI charged kaons are identified and tagged with the Ring Imaging Cherenkov counter (RICH), while $K_{s}^{0}$ are measured in their decay into charged pions.

The study of $\tau$ decays involving kaons provides a sensitive test for interactions beyond the minimal standard model. While in weak interactions these decays are strongly suppressed by the Cabibbo angle, this is generally not expected for new superweak interactions as predicted by many extensions. The decay $\tau \rightarrow K \nu$ is of special importance, since its branching ratio can be precisely calculated without any QCD corrections. The measurement of the $\tau$ decays into kaons is also needed for our understanding of other hadronic $\tau$ decays: The branching ratio of the decays $\tau \rightarrow h \nu$ and $\tau \rightarrow h \pi^{0} \nu$, where $h$ is either a pion or a kaon, have been measured very precisely. However, the contribution of kaons is much larger than the experimental uncertainty [1]. This contribution has to be known to compare the measurements with the standard model prediction.

\section{The DELPHI detector and the $\tau$ pair sample}

In the barrel region of the DELPHI detector [2], the charged particle tracks are reconstructed by a set of cylindrical tracking detectors. The Time Projection Chamber (TPC) is the main tracking device with a radius of the active area between $35 \mathrm{~cm}$ and $111 \mathrm{~cm}$. Track segments closer to the beam pipe are measured by the Inner Detector, which is a cylindrical drift chamber, and by the microvertex detector. The Barrel Ring Imaging Cherenkov (RICH) detector surrounds the TPC. Track measurements outside the RICH are provided by the Outer Detector.

Charged hadrons are identified with the Barrel RICH detector [3], which covers polar angles between $40^{\circ}$ and $140^{\circ}$, and employs a liquid and a gas radiator. The Cherenkov angle in the liquid radiator is already saturated for kaons of $3.5 \mathrm{GeV} / \mathrm{c}$, the threshold in $\tau$ decays at LEP energy. Therefore, in the $\tau$ analyses only the gas radiator is used, which allows $K / \pi$ separation at the $4 \sigma$ level up to $20 \mathrm{GeV} / \mathrm{c}$. The $40-\mathrm{cm}$ deep gas radiator volume behind the drift tubes is filled with $C_{5} F_{12}$. A set 288 parabolic mirrors focuses 
the Cherenkov photons from the gas radiator onto drift tubes. The projection has the form of a ring with a diameter proportional to the Cherenkov angle. Photons of wavelengths between 160 and $220 \mathrm{~nm}$ are in the drift tubes converted into electrons by a photosensitive additive, TMAE, in the drift gas. The photo-electrons are drifted towards an MWPC whose signals define the location of the photon conversion points with an accuracy of about $1 \mathrm{~mm}$ in all directions.

Electromagnetic showers are reconstructed by the High density Projection Chamber (HPC) in the barrel region. This detector covers the polar angular range between $41^{\circ}$ and $139^{\circ}$. The gas sampling technique used in the HPC provides a three-dimensional charge distribution measurement with high granularity.

The measurements presented here are based on the data collected in 1991 and 1992. The integrated luminosity corresponds to 48000 and $19000 \tau$ pairs for the $K_{s}^{0}$ and the $K^{ \pm}$analysis, respectively. The selection of $\tau$ pair events at LEP is straightforward. For all presented branching ratios, the uncertainties due to non- $\tau$ background are much smaller then the statistical errors.

\section{The decay $\tau \rightarrow K \nu$}

The DELPHI analysis of charged kaon production in $\tau$ decays has recently been published [4]. In the events selected for the $\tau \rightarrow K \nu$ analysis, one hemisphere has to contain a single charged particle with momentum between $4 \mathrm{GeV} / \mathrm{c}$ and $20 \mathrm{GeV} / \mathrm{c}$. Cuts on the energy deposit in the electromagnetic calorimeter are applied to remove decays with an electron or with $\pi^{0}$ in the final state. Finally, kaons are tagged on a track by track basis in conditions where their RICH response is well separated from the pion response. The kaon fraction is calculated from the number of events compatible with the kaon hypothesis, relative to those compatible with the $(\pi, \mu)$ hypothesis.

Two different techniques are used for kaon identification in the gas RICH. Between $4 \mathrm{GeV} / \mathrm{c}$ and $\sim 9 \mathrm{GeV} / \mathrm{c}$, kaons are below the threshold for producing Cherenkov photons, while pions and muons yield saturated angles. In this momentum range kaons are identified by "veto identif- cation", i.e. by requiring that no photo-electrons are associated to the particle. Above the kaon threshold of $\sim 9 \mathrm{GeV} / \mathrm{c}$ the Cherenkov angle is determined from the detected Cherenkov photons. This is called "ring identification", and allows kaon identification up to $20 \mathrm{GeV} / \mathrm{c}$. Together the two methods cover an important part of the phase space available in $\tau$ decays at LEP.

For reliable veto identification the yield of photo-electrons has to be well understood. The number of associated photo-electrons is defined as the number of detected photo-electrons in a $\pm 2.5 \sigma$ window around the saturated Cherenkov angle expected for muons and pions, $\theta_{(\pi, \mu)}$, which is $61 \mathrm{mrad}$. The expected number of photoelectrons is calculated for each track [3]. It depends on the momentum of the track and its position in the RICH, since the length of radiator passed by a particle increases with decreasing polar angle.

The distribution of the number of photoelectrons per track in dimuon events is shown in figure 1a. It has a mean value of 7.9. The measured and expected photo-electron distributions are in good agreement. This includes the bin with zero photo-electrons, which is used for the veto identification. In figures $1 \mathrm{~b}$ and $1 \mathrm{c}$ the same distributions are shown for tracks from single prong $\tau$ decays. In figure $1 \mathrm{~b}$ the particles have momenta between $20 \mathrm{GeV} / \mathrm{c}$ and $35 \mathrm{GeV} / \mathrm{c}$, where the mean expected number of photo-electrons is for all particles almost at its saturation value. Figure 1c shows the distribution for tracks selected for the exclusive analysis with momenta in the veto identification region. Here the expected number of photo-electrons is normalized to particles with observed photo-electrons. Particles without photo-electrons are tagged as kaons, the others belong to the $(\pi, \mu)$ class. The distribution of observed photo-electrons is identical, within statistical errors, for the three samples. The misidentification rate is the probability to observe no photo-electrons for a muon or pion. It was measured to be $(0.04 \pm 0.02) \%$ and $(0.09 \pm 0.09) \%$ for dimuon events and for high momentum tracks from $\tau$ decay, respectively. These numbers are compatible with Poisson statistics for the expected number of photo-electrons. 

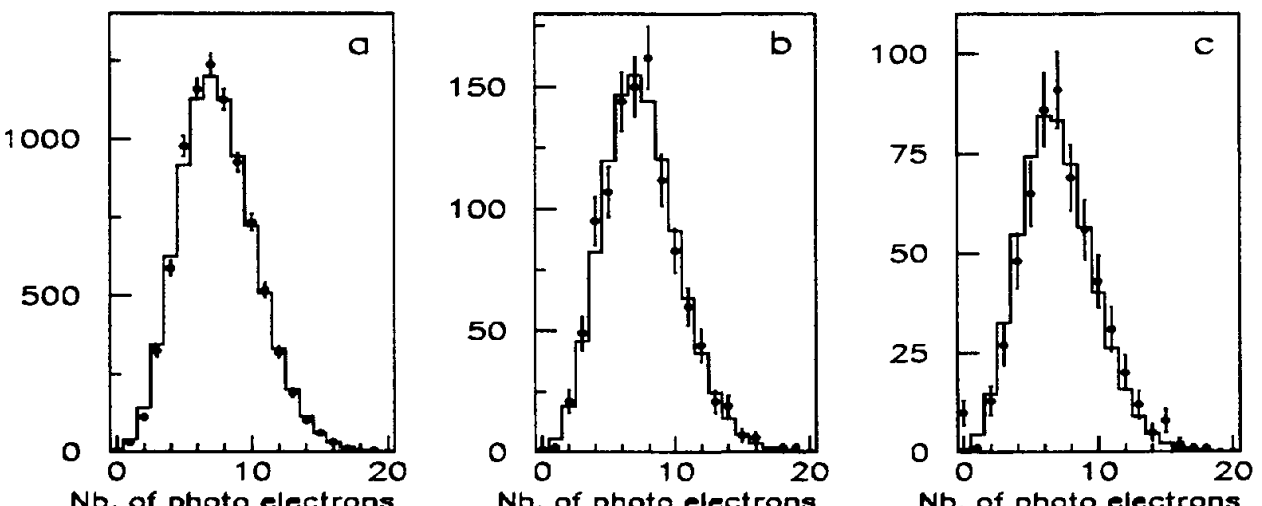

Nb. of photo electrons

Figure 1. Observed and expected number of photons per particle: (a) $Z \rightarrow \mu^{+} \mu^{-}$events; (b) high momentum particles from single prong $\tau$ decay; (c) particles from single prong $\tau$ decay in the veto region of kaon identification. The expected distribution (c) is for muons and pions only, the observed excess at zero photons is due to kaons.

Above threshold, the average Cherenkov angle $\theta_{m}$ for each track is determined. The detected photo-electrons are grouped into clusters. The angle $\theta_{m}$ is calculated as the weighted average of all photo-electrons in the cluster. An iterative procedure removes background hits and allows inclusion of new photons into the cluster. Photo-electrons with an individual Cherenkov angle within $\mathrm{a} \pm 2.5 \sigma$ window around $\theta_{m}$ are kept in the cluster. The final cluster has to contain at least two photons. The uncertainty on $\theta_{m}$ is $1.6 \mathrm{mrad}$ on average, and varies from track to track between $0.5 \mathrm{mrad}$ and $4 \mathrm{mrad}$. This is mainly due to the statistical fluctuations in the number of photo-electrons. The resolution function of $\theta_{m}$, defined as the difference between the measured and the expected Cherenkov angle divided by the expected error, is well described by a two-gaussian fit. The width of the main gaussian is $\sigma=1.01$, indicating that for most tracks the measured Cherenkov angle agrees with the expected value within the calculated error. Tails in the distribution, due to background and mis- alignments of mirrors, are accounted for with a second gaussian with a relative amplitude of 0.10 and width $\sigma=1.96$.

Figure 2 shows the measured Cherenkov angle $\theta_{m}$ as a function of particle momentum for the selected tracks. Also shown are the expected angles for $\mathrm{K}, \pi$ and $\mu$. Population of the kaon band is clearly observed. A particle with momentum above kaon threshold is tagged as a kaon if the measured Cherenkov angle is compatible with the kaon hypothesis within $\pm 2.5 \sigma$ and incompatible with the $(\pi, \mu)$ hypothesis, i.e. more than $4 \sigma$ below $\theta_{(\pi, \mu)}$. Similarly, a track was accepted in the $(\pi, \mu)$ class if the observed Cherenkov angle was compatible with $\theta_{(\pi, \mu)}$ and incompatible with $\theta_{K}$. Using these cuts the acceptance is similar for both classes. In figure 3 the acceptance is shown as a function of momentum. At momenta above $15 \mathrm{GeV} / \mathrm{c}$, the bands around the kaon and the $(\pi, \mu)$ hypotheses overlap, and the separation cut rejects an increasing number of tracks. At $20 \mathrm{GeV} / \mathrm{c}$ good $\mathrm{K}-\pi$ separation on a track by track basis is still possible for $\sim 10 \%$ of the cases. 


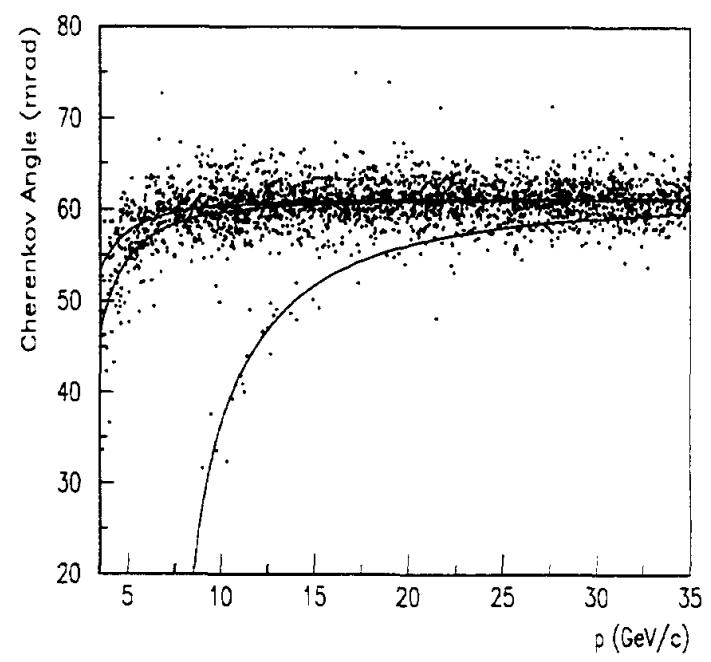

Figure 2. Measured Cherenkov angle versus momentum for single prong $\tau$ decays. The uncertainty varies from track to track between $0.5 \mathrm{mrad}$ and $4 \mathrm{mrad}$, depending on the number of photoelectrons. The expected angles for $K, \pi$ and $\mu$ are also shown.

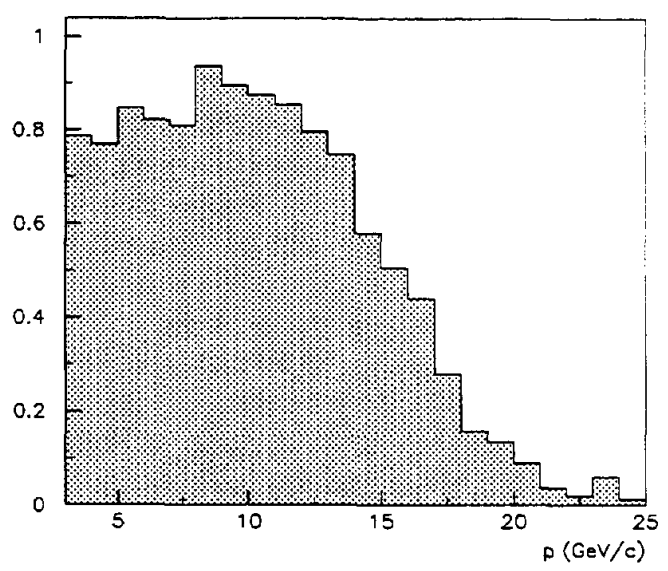

Figure 3. Acceptance for particle identification at the $4 \sigma$ level as a function of momentum
The branching ratio of the decay $\tau \rightarrow K \nu$ is obtained from the measured kaon fraction and from the results of a KORALZ Monte Carlo [5] simulation. The different steps are outlined in table 1. The first line gives the number of kaon candidates. The background from misidentification in the RICH is subtracted. The number of $\tau \rightarrow K^{*} \nu$ decays passing the cuts has been taken from the simulation. The contribution from each $\tau$ decay mode to the $(\pi, \mu)$ class has to be known to calculate the branching ratio. The KORALZ simulation is used to determine these contributions. The momentum dependent acceptance for RICH identification is applied to all tracks in the Monte Carlo sample that passed event selection cuts.

The branching ratio determined from the selected kaon sample is

$$
B R(\tau \rightarrow K \nu)=(0.85 \pm 0.18 \pm 0.03) \%
$$

The main sources to the systematic uncertainty are the composition of the event sample, the effciency of the different cuts and the RICH resolution function.

As a cross check the analysis is repeated for the veto and ring methods separately. Since both methods are applied to tracks between about $8 \mathrm{GeV} / \mathrm{c}$ and $10 \mathrm{GeV} / \mathrm{c}$, the splitting of the samples is done on a statistical basis. For this reason, the numbers of observed tracks in the $K$ and $(\pi, \mu)$ class are not integers. As can be seen in table 1 , the resulting branching ratios are in good agreement.

In the standard model, the branching ratio is predicted with negligible theoretical uncertainties. In particular the QCD corrections, parametrized by the decay constant $f_{K}$, can be taken from the $K \rightarrow \mu \nu$ decay. The calculation of the width $\Gamma(\tau \rightarrow K \nu)$ from the kaon decay involves only trivial phase space factors:

$$
B R_{T h}(\tau-K \nu)=(0.73 \pm 0.01) \%
$$

The error reflects the experimental uncertainties for the $\tau$ lifetime, the $K$ lifetime and the branching ratio of the decay $K \rightarrow \mu \nu$. The only theoretical uncertainty comes from QED radiative correction. These corrections amount to $0.9 \%[6]$ 
Table 1

Steps for the determination of $B R(\tau \rightarrow K \nu)$.

\begin{tabular}{|c|c|c|c|}
\hline & Combined & \multicolumn{1}{|c|}{ Veto } & \multicolumn{1}{c|}{ Ring } \\
\hline$K$ Candidates & $30.00 \pm 5.48$ & $10.15 \pm 3.19$ & $19.85 \pm 4.46$ \\
Misidentifications & $2.04 \pm 0.42$ & $0.80 \pm 0.23$ & $1.24 \pm 0.35$ \\
$K^{*}$ Background & $1.22 \pm 0.31$ & $0.17 \pm 0.04$ & $1.06 \pm 0.26$ \\
$K$ Signal & $26.74 \pm 5.49$ & $9.18 \pm 3.19$ & $17.56 \pm 4.48$ \\
$(\pi, \mu)$ Signal & 1316. & 555.20 & 760.80 \\
\hline$B R(\tau \rightarrow K \nu)[\%]$ & $0.85 \pm 0.18$ & $0.73 \pm 0.25$ & $0.94 \pm 0.25$ \\
\hline
\end{tabular}

and the uncertainty is small compared to the experimental errors.

Due to the precise theoretical prediction the measurement is sensitive to interactions beyond the standard model. The search for such interactions has become increasingly attractive. Many models attempting to unify the known interactions have been proposed. Most of these models predict additional superweak interactions. The couplings of these new interactions can be different for fermions of different generations, e.g. through mixing angles or mass dependent coupling constants. To derive limits on such interactions, we write the lagrangian for the semileptonic decays in a general form

$$
\frac{G}{\sqrt{2}}\left[g_{s} g_{l}(\bar{u} \Gamma s)(\bar{l} \Gamma \nu)+g_{s}^{\prime} g_{l}^{\prime}\left(\bar{u} \Gamma^{\prime} s\right)\left(\bar{l} \Gamma^{\prime} \nu\right)\right]
$$

Here, the first term is due to the normal V-A weak current and the second one is due to possible new interactions. The subscript $l$ in the coupling constants stands for the coupling to leptonneutrino and $s$ for the coupling to a $u \bar{s}$ quark pair. The corresponding coupling to $u \bar{d}$ is defined as $g_{d}$. The coupling constants are given in the standard model:

$$
\begin{aligned}
g_{d}=\cos \theta_{c} \quad g_{s}=\sin \theta_{c} & g_{\tau}=g_{\mu}=g_{e}=1 \\
g_{d}^{\prime} & =g_{s}^{\prime}=g_{t}^{\prime}=0 .
\end{aligned}
$$

The test of universality in the standard weak interaction $\left(g_{\tau}=g_{\mu}=g_{e}\right)$ is best done with the purely leptonic $\tau$ decays [7]. Here I concentrate on additional interactions. For simplicity I will only consider interactions mediated by a spin 1 boson. Assuming the standard model values for the normal weak couplings, the $\tau$ decay rate is

$\Gamma(\tau \rightarrow K \nu)=$
Table 2

Limits on new interactions beyond standard model in units of the Fermi coupling

\begin{tabular}{|c|c|}
\hline Decays & Limit \\
\hline$\frac{\Gamma(\pi \rightarrow e \nu)}{\Gamma(\pi-\mu \nu)}$ & $g_{d}^{\prime}\left(g_{\mu}^{\prime}-g_{e}^{\prime}\right)<0.004$ \\
$\frac{\Gamma(\tau-e \nu \nu)}{\Gamma(\mu-e \nu \nu)}$ & $g_{e}^{\prime}\left(g_{\tau}^{\prime}-g_{\mu}^{\prime}\right)<0.010$ \\
$\frac{\Gamma(\tau \rightarrow \pi \nu)}{\Gamma(\pi \rightarrow \mu \nu)}$ & $g_{d}^{\prime}\left(g_{\tau}^{\prime}-g_{\mu}^{\prime}\right)<0.018$ \\
$\frac{\Gamma(\tau \rightarrow K \nu)}{\Gamma(K-\mu \nu)}$ & $g_{s}^{\prime}\left(g_{\tau}^{\prime}-g_{\mu}^{\prime}\right)<0.018$ \\
\hline
\end{tabular}

$$
\frac{G^{2} f_{K}^{2}}{8 \pi}\left(\sin \theta_{c}+g_{s}^{\prime} g_{\tau}^{\prime}\right) m_{\tau}^{3}\left[1-\frac{m_{K}^{2}}{m_{\tau}^{2}}\right]^{2}
$$

Since the decay constant $f_{K}$ cannot be calculated reliably, we have to compare the $\tau$ decay rate with the kaon decay rate:

$$
\begin{aligned}
& \frac{\Gamma(\tau \rightarrow K \nu)}{\Gamma(K \rightarrow \mu \nu)}= \\
& \quad\left[\sin \theta_{c}+2\left(g_{s}^{\prime}\left(g_{\tau}^{\prime}-g_{\mu}^{\prime}\right)\right)\right] \cdot P S
\end{aligned}
$$

The factor $P S$ corrects for the difference in the available phase space and depends only on the masses of the particles involved. This method is sensitive to differences in the coupling constants of new interactions, i.e. to lepton universality violating interactions.

A list of limits derived from different leptonic decays is given in table 2 . For each ratio of decay rates an equation analogous to eq.(6) can be written. The numbers in the table are based on the new world averages for $\tau$ decays presented at this conference $[1],[7]$, and the recent measurements 
of the decay $\pi \rightarrow e \nu[8]$, which gives the lowest limit for new interactions. However, with the natural assumption $g_{\tau}^{\prime} \gg g_{\mu}^{\prime} \gg g_{e}^{\prime}$, the semileptonic $\tau$ decays are probably more sensitive. The decay $\tau \rightarrow K \nu$ plays a central role in the search for new interactions. Because is suppressed by a factor $\sin \theta_{c}$, it is intrinsically more sensitive to new interactions as compared to the corresponding decay into $\pi$. It also leads to a limit which does not depend on coupling to fermions of the first generation, where the effect of lepton universality violating interactions might be very small. From the experimental point of view, the measured branching ratio is needed to derive the branching ratio of the decay $\tau \rightarrow \pi \nu$ from the measured $B R(\tau \rightarrow h \nu)$.

The charged kaon identification in the DELPHI $\mathrm{RICH}$ was also used to measure the inclusive kaon production in $\tau$ decays [4]. The method is similar to the one used for the exclusive decay $\tau \rightarrow K \nu$. The results are

$$
\begin{gathered}
B R(\tau \rightarrow K \nu \geq 0 \text { neutrals })=(1.54 \pm 0.24) \% \\
B R(\tau \rightarrow K \nu \geq 1 \text { neutrals })=(0.64 \pm 0.24) \%,
\end{gathered}
$$

where the measured exclusive branching ratio (1) is used to separate on a statistical basis the decays with and without neutrals.

\section{The decay $\tau \rightarrow K^{*} \nu$}

In DELPHI the $\tau$ decay into $K^{*}$ is studied in the two modes $K^{* \pm} \rightarrow K^{ \pm} \pi^{0}$ and $K^{* \pm} \rightarrow \pi^{ \pm} K_{s}^{0}$. In both analyses domination of the final states by the $K^{*}(892)$ resonance is assumed.

The $K_{s}^{0}$ are detected by reconstruction of the secondary vertex $V^{0}$ from the decay $K_{s}^{0} \rightarrow \pi^{+} \pi^{-}$. In $\tau$ decays with more than one reconstructed track, pairs of opposite charged tracks are formed. For each pair a 3-dimensional secondary vertex is fitted. Only fits with a $\chi^{2}$ probability of $>0.001$ are kept as $K_{s}^{0}$ candidates. Furthermore, the first measured point on each of the two tracks has to be located behind the reconstructed vertex, but not more than $30 \mathrm{~cm}$. The angle between the momentum of the candidate and the line of flight (connecting the beam spot with the vertex) has to be less than $0.6^{\circ}$. To reject $\gamma$ conversions and 3 -prong $\tau$ decays, the transverse momentum of

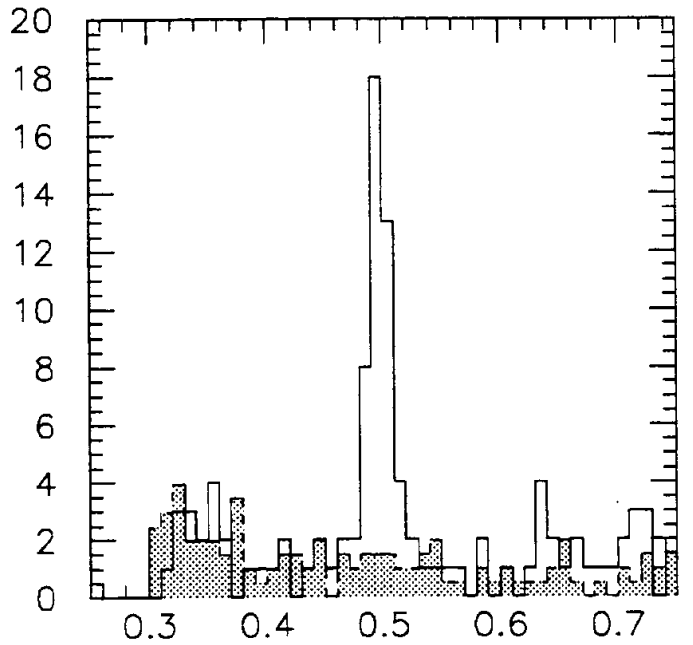

Figure 4. The $\left(\pi^{+} \pi^{-}\right)$mass spectrum of the $K_{s}^{0}$ candidates. The background expected from Monte Carlo simulation is also shown.

the tracks w.r.t. to the $V^{0}$ has to be larger than $60 \mathrm{MeV} / \mathrm{c}$ and its decay distance at least $3 \mathrm{~cm}$. The $\left(\pi^{+} \pi^{-}\right)$mass spectrum of the $V^{0}$ sample is shown in figure 4 . The observed background is in good agreement with the expectation from a full KORALZ simulation, which is shown as a shaded histogram.

A fit to the mass spectrum yields $(42.0 \pm 7.3)$ events in the $K_{s}^{0}$ peak. The detection efficiency for $K_{s}^{0} \rightarrow \pi^{+} \pi^{-}$in $\tau$ decays is derived from Monte Carlo simulation and amounts to $(17.4 \pm 1.4) \%$. The preliminary result for the inclusive $K_{s}^{0}$ branching ratio is

$B R\left(\tau \rightarrow K_{s}^{0} X^{ \pm} \nu\right)=(0.99 \pm 0.19) \%$.

For the measurement of $B R\left(\tau \rightarrow K^{*} \nu\right)$ secondary vertices $V^{0}$ are selected with a measured $\left(\pi^{+} \pi^{-}\right)$mass between $457 \mathrm{MeV}$ and $537 \mathrm{MeV}$. These $K_{s}^{0}$ candidates are combined with a third charged track $h^{ \pm}$in the same hemisphere. The resulting $V^{0} h^{ \pm}$mass spectrum is shown in figure 5. Again, the shaded area indicates the back- 


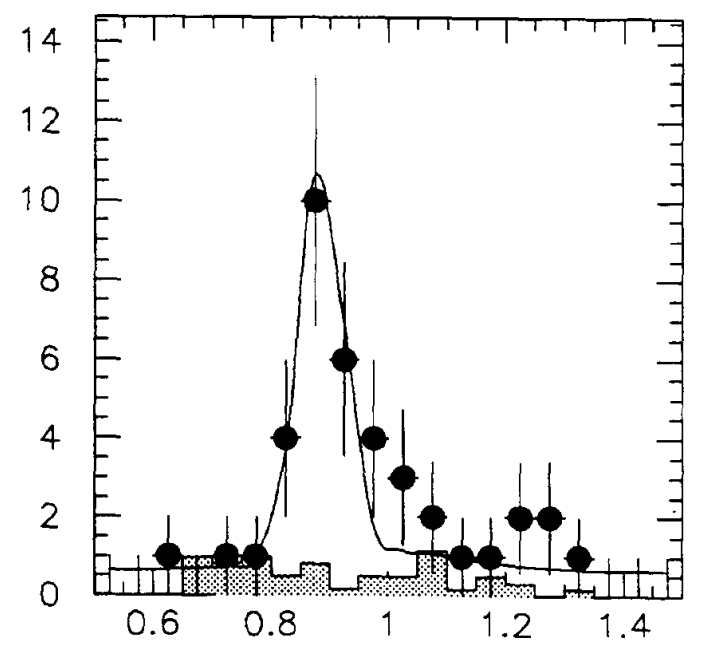

Figure 5. The $\left(h^{ \pm} \pi^{+} \pi^{-}\right)$mass spectrum. The background expected from Monte Carlo simulation is also shown.

ground expected from the KORALZ simulation. The number of $K^{*}, 22.7 \pm 5.2$, is obtained from a fit the mass spectrum, and the detection efficiency from the KORALZ simulation. The preliminary result for the branching ratio

$B R\left(\tau \rightarrow K^{*} \nu\right)=(1.74 \pm 0.42) \%$

implies that about $60 \%$ of the $K_{s}^{0}$ produced in $\tau$ decays are due to this exclusive decay.

The $K^{*}$ measurement in the channel $K^{ \pm} \pi^{0}[4]$ is a supplement of the inclusive kaon analysis described in the previous section. The kaons are identified in the $\mathrm{RICH}$, and tagged with very loose cuts. The $\pi^{0}$ 's are reconstructed in the electromagnetic calorimeter, as a single cluster with energy above $5 \mathrm{GeV}$, or as two clusters with invariant mass of less than $500 \mathrm{MeV}$. In the latter case, the energy of each cluster must be lower than $10 \mathrm{GeV}$, and the sum must exceed $2 \mathrm{GeV}$. Decays with additional photons in the hemisphere are rejected. $\mathrm{A}$ fit to the $K^{ \pm} \pi^{0}$ mass spectrum yields $15 \pm 5$ events under the $K^{*}$ peak. Similarly, charged pions are selected and combined with a $\pi^{0}$, and a total of $484 \pm 36 \rho$ decays are obtained. With the measured branching ratio of the decay $\tau \rightarrow \rho \nu$ we get

$B R\left(\tau \rightarrow K^{*} \nu\right)=(1.71 \pm 0.69) \%$,

which is in excellent agreement with the result from the $K_{s}^{0}$ channel. The combined result is

$B R\left(\tau \rightarrow K^{*} \nu\right)=(1.73 \pm 0.36) \%$.

To calculate the branching ratio in the standard model, the decay constant $f_{K}$ * has to be known. Unlike the constant $f_{K}$ discussed in the previous section, $f_{K}$. cannot be measured in an other decay, but must be calculated using QCD. A simple estimate is obtained if the $\pi \pi$ and the $K \pi$ final states are dominated by the $\rho(770)$ and the $K^{*}(892)$ resonances, respectively. In this case, the relation

$\frac{f_{K^{*}}^{2}}{m_{K^{*}}^{2}}=\frac{f_{\rho}^{2}}{m_{\rho}^{2}}$

can be derived from the Das-Mathur-Okubo sum rule, which describes the breaking of the $S U(3) \times S U(3)$ symmetry. The resulting prediction $B R\left(\tau \rightarrow K^{*} \nu\right) \simeq 1.2 \%$ is consistent with the measured branching ratio.

\section{Summary}

Several exclusive and inclusive branching ratios of $\tau$ decays with $K^{ \pm}$and $K_{s}^{0}$ in the final state have been measured. All results agree with the standard model prediction. Due to the precise theoretical prediction, the measured branching ratio of the exclusive decay $\tau \rightarrow K \nu$ is used to set stringent limits on new interactions which violate $\tau-\mu-e$ universality.

The measurements of the branching ratios involving charged kaons make use of the DELPHI Ring Imaging Cherenkov Counter. Kaons are tagged on a track by track basis with very low background. This technique is complementary to statistical $K-\pi$ separation, used in most other experiments. The uncertainties are dominated by the statistical errors. The small systematic uncertainties and the large sample of $\tau$ pairs already 
taken in 1993 and 1994 promise a fast progress in our understanding of Cabibbo suppressed $\tau$ decays.

I would like to thank all my DELPHI colleagues, who contributed to the achievement of the results presented here. I am especially grateful to Dick Holthuizen, Bert Koene, Francesco Navarria and Luc Pape for many stimulating discussions.

\section{REFERENCES}

1. B.Heltsley, Contribution to this Conference

2. P.Aarnio et al. Nucl. Instr. and Meth. in Phys. Res. A303 (1991), 233

3. E.Anassontzis et al. Nucl. Instr. and Meth. A323(1992), 351

4. P.Abreu et al. Phys.Lett. 334B (1994), 435

5. S.Jadach and Z.Was, Comput. Phys. Commun. 36 (1985) 191

6. R.Decker and M.Finkemeier, Karlsruhe University, TTP94-5 (1994)

7. M.Davier, Contribution to this Conference

8. D.I.Britton et al. Phys.Rev.Lett. 68 (1992) 3000; G.Czapek et al. Phys.Rev.Lett. 70 (1993) 17 